\title{
Application of levator veli palatini retropositioning combined with Buccinator myomucosal island flap for congenital cleft palate
}

\author{
Y.J. WAN, H.C.ZHANG, Y.ZHANG, Y.S.CHENG, Y.ZHANG and C. WANG \\ Department of Stomatology, First People's Hospital of Xuzhou City, Xuzhou, Jiangsu 221002, P.R. China
}

Received October 30, 2015; Accepted April 28, 2016

DOI: $10.3892 / \mathrm{etm} .2016 .3666$

\begin{abstract}
Congenital cleft palate causes a serious obstacle to children with regard to language and eating function. The aim of the current study was to examine the clinical application of a type of palatoplasty that has a reduced impact on the maxillary growth and good function in velopharyngeal competence. A total of 37 patients with cleft palate were treated with levator veli palatini retropositioning combined with Buccinator myomucosal island flap. The patients were successfully treated in the first phase and were followed up for 1-3 years. Speech intelligibility was satisfactory and no fistula occurred. In conclusion, the results suggested that levator veli palatini retropositioning combined with the Buccinator myomucosal island flap may restore normal anatomic structure and location of the levator veli palatini, obtain good velopharyngeal competence, and decrease the incidence rate thereof. Thus, levator veli palatini retropositioning combined with the Buccinator myomucosal island flap is a functional procedure for cleft palate repair.
\end{abstract}

\section{Introduction}

Congenital cleft palate poses a serious obstacle to children in language and eating function (1). Good speech effect following surgery is particularly important for children with regard to mental development and self-confidence in social communication. Previous efforts of clinicians and phonetic experts were focused on children obtaining normal speech effect. Surgical methods have advanced over the previous 100 years, and currently initial simple closed fracture to functional repair are utilized (2). In recent years, the procedure of levator veli palatini retropositioning for cleft palate has become mainstream, but the higher incidence of palatal fistula has had an impact

Correspondence to: Dr Hong-Chuang Zhang, Department of Stomatology, First People's Hospital of Xuzhou City, 19 Zhongshan North Road, Xuzhou, Jiangsu 221002, P.R. China

E-mail: hczhang21@163.com

Key words: cleft palate, levator veli palatini retropositioning, Buccinator myomucosal island flap, velopharyngeal competence on clinical promotion. Between 2006 and 2010, 37 patients with cleft palate were treated with levator veli palatini retropositioning combined with Buccinator myomucosal island (3). The treatment was succesful and follow up of patients for 1-3 years revealed that speech intelligibility was satisfactory. No postoperative palatal fistula occurred. At present, levator veli palatini retropositioning combined with Buccinator myomucosal island flap has gradually replaced traditional methods and has become a conventional surgical method for cleft palate repair.

\section{Patients and methods}

Clinical materials. In total, 37 patients (21 males and 16 females) with non-syndrome cleft palate, were included in the study. The age range of the patients was 5-9 years. There were 26 cases with incomplete cleft palate, and 11 cases with one side complete cleft palate, The children with screening were well-developed, no other concurrent disease occurred, hemoglobin was $>100 \mathrm{~g} / 1$ and no respiratory tract infections were observed over a period of $\sim 2$ weeks. On the two sides of the roots of the uvula point, the slit width was $9-25 \mathrm{~mm}$. Sommerlad palatine velum levator muscle reconstruction combined with island-shaped buccal mucosa muscle flap surgery was applied for patients with a cleft palate.

This study was approved by the ethics committee of the First People's Hospital of Xuzhou City (Jiangsu, China). Written informed consent was obtained from all the participants before the study.

Surgical instruments. Cleft palate routine surgical instruments and 3.0x portable operation microscope (Heine, HR2.5x, Berlin, Germany) were used. The head of an electric knife (Shuyou Company, SY-IIB(N)-2; Hangzhou, Zhejiang, China) was connected to a ventricle drainage tube (only $5 \mathrm{~mm}$ outside) to avoid burns on the surrounding tissue of the mouth. A cleft palate gag was used to keep the lips open, exposing the operative field. Absorbable sutures were used in stitching.

Anesthesia method. Oral endotracheal intubation and general anesthesia were administered. A quantity of 1/100,000 adrenaline saline was used in the operation area of infiltration anesthesia to reduce intraoperative blood and to avoid the unclear vision field on muscle dissection. 
Surgical technique

Formation of the mucoperiosteal flap by opening the crack edge. Fifteen blades were used to open the mouth of the periosteum layer in the partial side of oral mucosa of the fractured edge, in order that the back part of the oral mucosa be used as nasal mucosa to ease the tension thereof. The two sides of the notch converted at midline and upwardly extended to the hard palate slightly. The buccal mucosa flap contained glands that were turned over to retain palatal muscles in the side of the nasal mucosa.

Loosening of large nerve and vascular bundle. The vascular bundle of the palatine nerve was revealed in the posterior border of the hard palate as slightly lateral, the nerve hook was used to open the mouth side tissue flap, a part of the neurovascular bundle was under blunt dissection around the greater palatine foramen, as far as possible from the wingless uncinate process, and lateral muscle bundles were retained with the open eustachian tube function.

Isolation and suture of side of the nasal mucosa. Nasal mucosa were initially isolated by the interrupted suture of the fractured edge, and the knots were left in the nasal side, thus the mucosa was in a slightly tightened state. The palatine aponeurosis and muscle tissue attached to the hard palate posterior were blocked along the trailing edge of the hard palate and nasal spine by kitchen knives and the integrity of the nasal mucosa was retained.

Free reconstruction of levator veli palatine. Palatoglossus and pharyngeal jaw muscle fibers were re-arranged, turned over and distracted, and cylindrical palatine velum muscle fibers were visible. There was a boundary between levator veli palatini muscle and nasal mucosa. Levator veli palatini muscle was freed along the boundary, and intact sarcolemma of the levator veli palatini muscle was retained. The levator veli palatini muscle was rotated freely to the midline under the stretching on the confluence of fascicles on the other side in $2 / 3$ of the soft palate. Subsequently, the levator veli palatini muscle was returned and was closed with interrupted sutures in the middle and under part of soft palate.

Preparation of the Buccinator myomucosal island flap. The anterior border of the Buccinator myomucosal island flap was slightly posterior to angular, the posterior border was located outside the wing jaw ligament (the anterior portion of the $5 \mathrm{~mm}$ ), which provided a sufficient width of the mucosal tunnel, and part of the mucosa was severed to form the island flap. The upper border of the island flap was located below the opening of the parotid gland of $2 \mathrm{~mm}$ to avoid the damage on pipes under suture. The appropriate valve width used was $10-15 \mathrm{~mm}$ to form a tongue-shaped flap similar to the hard palate fissure as far as possible. The mucous layer in front of the pterygomandibular ligament was incised, and was dissected until the wound edge of the hard palate junction by tissue scissors in the submucosal to form a tunnel under the mucous membrane. The facial artery after buccal branches, which protected the domination of the pedicle, were dissected on a buccal fat pad surface and attention was paid to hemostasis. The wound was directly sutured.
Suture of Buccinator myomucosal island flap in the oral mucosa. The hard palate incision margin was freed $\sim 2 \mathrm{~mm}$, one side of the Buccinator myomucosal island flap was used to cover the wounds on the same side, and the remaining wounds were covered by the other side of the buccal mucosa muscle flap. The mattress suture through the nasal mucosa, muscle, and oral mucosa was applied. However, mattress suture was not usually applied as the recovery degree after healing between oral and nasal mucosa was satisfactory following suture under no tension. Following the interrupted suture on oral mucosa layer by absorbable suture, hemostatic gauze was used to stem any bleeding.

\section{Operation effect}

Incidence of anesthesia and surgical complications. There were no complications, such as anesthesia accidents. Additionally, no postoperative wound infection or fistula were reported.

Intraoperative blood loss. The average operation time was $95 \mathrm{~min}$, the amount of bleeding was $\sim 35 \mathrm{ml}$, and the average was 20-60 ml. No blood transfusion was required.

Treatment effect evaluation. Patients who underwent operation of levator veli palatini retropositioning combined with Buccinator myomucosal island flap had postoperative and regular follow up for 1-3 years. One month after the operation, the palate was healed normally, and no scar or palatal fistula occurred. The activities of soft palate and lateral pharyngeal wall and recovery degree of cheek wound were good. Voice teacher training, pharyngeal cavity angiography, and nasopharyngeal fiberscope, were applied, and the results showed that speech intelligibility, high nasal and nasal air leak were significantly improved. Ten children were able to cooperate with us, and pharyngeal cavity angiography showed that the closure rate of the pharyngeal cavity was $70 \%$ after operation.

\section{Results and Discussion}

General. The main damage to children with congenital cleft palate were malnutrition and growth retardation, especially language dysfunction caused by feeding difficulties, which seriously interfere with a child's normal social interaction and mental health formation (4). The purpose of modern cleft palate repair lies, not only on the close fractures, but more importantly on the reconstruction of the soft palate muscle function, to reach velopharyngeal closure to obtain a good voice, and to minimize the inhibition of maxillary growth by hard palate bone surface exposure at the same time (5). Conducting operation of levator veli palatine reconstruction dissected the ectopic muscle layer of levator veli palatine and reconstructed the levator sling, did not expose or exposed less the bone surface of the hard palate as far as possible, and sutured wounds with the application of absorbable suture. Therefore, it had advantages such as small trauma, improved language, little effect on maxillary growth and development, and postoperative rapid recovery (6). However, clinical reports showed that because of the emphasis on the avoidance of relaxation incision of this method, the incidence of palatal fistula was higher (7), which limited the clinical application for the children with broader cleft palate. 
Significance of levator veli palatine reconstruction. In all the soft palate muscles associated with speech, levator veli palatine was the main functional muscle (8). Levator veli palatine reconstruction reset palatine velum muscle fibers from the trailing edge of the hard palate and nasal crest to the normal position through the operation. In addition, the muscle raised the soft palate under pronunciation to elevate it, after which the soft palate and the posterior pharyngeal wall were wellcontacted with the other side, resulting in good velopharyngeal closure. Thus, the physiological function and effect on palatal pharyngeal closing of levator veli palatine were restored (5).

Surgical techniques and experiences of levator veli palatini retropositioning combined with Buccinator myomucosal island flap

Soft operation and the control of intraoperative bleeding. As the patients were of a young age, the oral cavity volume was small. Thus, the effect of anesthesia, intubation, and the visual field of operation was limited. Furthermore, the organization was more delicate, there were increased sites of infiltration blood, and anatomic landmark was not sufficiently obvious, requiring care to minimize damage to muscle when separating the levator veli palatine during the operation. Under optimal conditions, a microscope should be applied when soft palate muscles were dissected to achieve full anatomy and less damage. Local bleeding should be reduced as far as possible, multi-point injection of adrenaline saline on the soft palate should be given before incision, and timely electric coagulation bleeding for hemostatis should be used.

Anatomy, separation, and suture of levator veli palatine. The precise anatomy and paraposition suture were crucial in restoring the normal anatomy and position of levator veli palatine and in reconstructing the function of the palate. The 'light blue' nasal mucosa under a light microscope showed the process of muscle dissection and separation, indicating that all of the muscle and tendon tissue was thoroughly stripped out from the nasal mucosa (9). Concerning the process of anatomy, large ruptures should be avoided on nasal mucosa as far as possible. If the rupture is large, suture should be applied to avoid refissure.

Preparation and processing bilateral buccal mucosa flap and pedicle. As previously reported in literature, buccal mucosa flap was an axial pattern flap with large randomness of the ratio of length and width. However, the design principles included techniques that would result in no damage to the parotid duct opening and the healing wound would close easily and directly (10). Owing to innervation, rich blood supply, and strong healing ability, the buccal mucosa flap did not atrophy. We considered the appropriate thickness of the buccal mucosa flap to be $3 \mathrm{~mm}$, with the buccinator attachment occurring in the front end of the flap. Anatomy should then be operated on along the deep level of buccinators in a backward manner in order that damage in fascia be avoided. The blood supply of buccal mucosa flap with back pedicle originated from the facial artery (11). Correct intraoperative isolation levels would not damage the facial nerve and posterior buccal branch of facial artery on the deep position of buccinator.
At present, scholars generally advocate that relaxing incisions should not be employed in cleft palate surgery in order to avoid inducing scar tissue formation, which had a negative effect on the development of jaw and maxillofacial region (12). Large tension was the main reason for palatal fistula after traditional operation for cleft palate, i.e., no relaxation incision. The main reason for postoperative fistula is excessive tension when not using relaxation incision in traditional cleft palate operations. Additionally, the island buccal mucosal flap can effectively relieve the tension of oral mucosa, and avoid the influence of bare bone in the face of maxillary development.

In conclusion, the results of the present study have shown that levator veli palatini retropositioning was superior to traditional operation in surgical trauma, intraoperative bleeding and voice recovery, and it was more in concordance with the principle of cleft palate repair. Combined island buccal mucosa flap in the same period was more effective in relieving the tension of the oral mucosa, reducing the incidence of the fistula and promoting the recovery of the function of palate closure. However, since the application time of the operation was limited, it had a high requirement in surgical skills, and intraoperative trauma was slightly larger than traditional surgery. To resolve such issues the option of surgical indications and long-term efficacy of levator veli palatini retropositioning combined with Buccinator myomucosal island flap should be further studied to confirm the results of the present study.

\section{References}

1. Ying B, Ye W and Li Z: Tongue musculomucosal flap for soft palate reconstruction in patients with OSAHS - clinical experience in technical strategy. Eur Rev Med Pharmacol Sci 17: 1963-1966, 2013.

2. Eichenberg B, Punjabi AP and D'Antonio L: Long term outcome analysis of two treatment methods for cleft palate: Combined levator retropositioning and pharyngeal flap versus doubleopposing Z-plasty. Cleft Palate Craniofac J 37: 326, 2000.

3. Fisher JC and Edgerton MT: Combined use of levator retrodisplacement and pharyngeal flap for congenital palate insufficiency. Cleft Palate J 12: 270-273, 1975.

4. Panetta NJ, Gupta DM, Slater BJ, Kwan MD, Liu KJ and Longaker MT: Tissue engineering in cleft palate and other congenital malformations. Pediatr Res 63: 545-551, 2008.

5. Karsten A, Larson M and Larson O: Dental occlusion after Veau-Wardill-Kilner versus minimal incision technique repair of isolated clefts of the hard and soft palate. Cleft Palate Craniofac J 40: 504-510, 2003.

6. Lu Y, Shi B, Zheng Q, Li Y and Meng T: The application of Sommerlad levator veli palatini muscle reconstruction on cleft palate repair. J Clin Stomatol 22: 233-236, 2006.

7. Emory RE Jr, Clay RP, Bite U and Jackson IT: Fistula formation and repair after palatal closure: an institutional perspective. Plast Reconstr Surg 99: 1535-1538, 1997.

8. Lv Z, Xu X and Du J: The comparative study of 3 kinds of palatoplasty with subjective and objective evaluation method. J Oral Sci Res 22: 195-196, 2006.

9. Wu AQ, Li JY, Nie ZM, Zhang J, Ma Z, Chu XX, Li ZD, Jie BJ and Guo BF: Applied anatomy of buccal mucosa flap. Chin J Clin Anat 16: 31-33, 1998.

10. Zhao Z, Li S, Yan Y, Li Y, Yang M, Mu L, Huang W, Liu Y, Zhai $\mathrm{H}$, Jin J, et al: New buccinator myomucosal island flap: anatomic study and clinical application. Plast Reconstr Surg 104: 55-64, 1999.

11. Sommerlad BC: A technique for cleft palate repair. Plast Reconstr Surg 112: 1542-1548, 2003.

12. Wijdeveld MG, Maltha JC, Grupping EM, De Jonge J and Kuijpers-Jagtman AM: A histological study of tissue response to simulated cleft palate surgery at different ages in beagle dogs. Arch Oral Biol 36:837-843, 1991. 